\title{
Video Article \\ Intraductal Injection for Localized Drug Delivery to the Mouse Mammary Gland
}

\author{
Silva Krause ${ }^{1}$, Amy Brock ${ }^{2}$, Donald E. Ingber ${ }^{1,2,3}$ \\ ${ }^{1}$ Vascular Biology Program, Department of Surgery, Boston Children's Hospital and Harvard Medical School \\ ${ }^{2}$ Wyss Institute for Biologically Inspired Engineering, Harvard University \\ ${ }^{3}$ Harvard School of Engineering and Applied Sciences
}

URL: https://www.jove.com/video/50692

DOI: doi:10.3791/50692

Keywords: Developmental Biology, Issue 80, Mammary Glands, Animal, Drug Administration Routes, intraductal injection, local drug delivery, siRNA

Date Published: 10/4/2013

Citation: Krause, S., Brock, A., Ingber, D.E. Intraductal Injection for Localized Drug Delivery to the Mouse Mammary Gland. J. Vis. Exp. (80), e50692, doi:10.3791/50692 (2013)

\section{Abstract}

Herein we describe a protocol to deliver various reagents to the mouse mammary gland via intraductal injections. Localized drug delivery and knock-down of genes within the mammary epithelium has been difficult to achieve due to the lack of appropriate targeting molecules that are independent of developmental stages such as pregnancy and lactation. Herein, we describe a technique for localized delivery of reagents to the mammary gland at any stage in adulthood via intraductal injection into the nipples of mice. The injections can be performed on live mice, under anesthesia, and allow for a non-invasive and localized drug delivery to the mammary gland. Furthermore, the injections can be repeated over several months without damaging the nipple. Vital dyes such as Evans Blue are very helpful to learn the technique. Upon intraductal injection of the blue dye, the entire ductal tree becomes visible to the eye. Furthermore, fluorescently labeled reagents also allow for visualization and distribution within the mammary gland. This technique is adaptable for a variety of compounds including siRNA, chemotherapeutic agents, and small molecules.

\section{Video Link}

The video component of this article can be found at https://www.jove.com/video/50692/

\section{Introduction}

The mouse mammary gland is composed of a complex ductal-alveolar tree that converges into a central duct at the nipple. In mice as well as humans, the majority of breast tumors originate in the epithelial cells lining the milk ducts. Current available treatments include intravenous chemotherapy, radiation, and surgery. Localized intraductal treatments have been explored ${ }^{1-3}$ and offer significant advantages in terms of reduced toxicity and side effects. However, several challenges remain before these can be applied more widely. The ability to perform comparable procedures in the mouse mammary gland facilitates the characterization of intraductal interventions. Here we present a non-invasive method for the delivery of reagents directly into the mouse mammary duct.

Intraductal delivery of chemotherapeutic agents in both breast cancer patients and mouse models has previously been shown to be highly effective with no evidence of systemic toxicity or long-term histopathological changes ${ }^{3-6}$. Furthermore, tumor cell injection and lentiviral vector delivery of oncogenes have been shown previously to be feasible via intraductal injection ${ }^{7-10}$.

In addition to drug delivery, this procedure is useful for local silencing of mammary gland genes through intraductal injection of siRNA. Injection of small volumes of siRNA solution is effective without altering the anatomy and physiology of the mammary gland. In our laboratory we have recently shown successful silencing of key mediator genes in transgenic mice that spontaneously develop mammary tumors resulting in prevention of tumor progression (Brock et al., unpublished). We have been able to repeat biweekly injections into the same nipples up to 7 times in 2-week intervals with no visible tissue damage or toxicity.

Intraductal injections can be timed in an adult mouse to address developmentally-specific events relevant to mammary gland development during pregnancy, lactation, and involution or to target later stages during tumor development and progression. Virgin females as young as 8 weeks of age have been injected non-invasively in our laboratory.

\section{Protocol}

\section{Preparation of Injection Solution}

1. Prepare $0.2 \%$ Evans blue dye in phosphate buffered saline (PBS) or other aqueous solution of interest. This vital dye is useful for assessing the success of intraductal injections and is recommended as a visualization aid when developing expertise in the technique. 
The volume required will depend on the number and location of the glands to be injected. All 10 mammary glands of female mice can be injected but due to the smaller gland size, gland pairs 1 and 5 are typically injected with $10 \mu$ of solution. All other mammary pairs are injected with $20 \mu$. These volumes are sufficient to fill the entire ductal tree.

\section{Preoperative Preparation}

1. Record the body weight of each mouse. As with all preclinical studies, animal weights should be monitored regularly (twice a week or greater) to assess potential toxicity.

2. Anesthetize the mouse using an isoflurane chamber and apply eye lubricant. During the procedure mice will continue to be anesthetized using inhaled $2-4 \%$ concentration of isoflurane in oxygen via a nose cone. Carefully monitor the mouse for changes in respiratory rate, adjusting the level of isoflurane accordingly.

3. Inject meloxicam $(5-10 \mathrm{mg} / \mathrm{kg})$ subcutaneously prior to the procedure as analgesia.

4. Apply an over-the-counter hair removal cream to the nipple area. Wait $5 \mathrm{~min}$ and gently remove loose hair with a cotton-tip applicator using a circular motion. Remove the cream using damp paper towels wetted with warm water. Shaving is not recommended due to the risk of damaging the nipples.

5. Secure the mouse under the stereoscope by gently taping down the extremities.

6. Clean injection sites with alcohol swabs.

\section{Intraductal injection}

1. Locate appropriate nipples to be injected under the stereoscope. Use fine micro-dissecting tweezers to remove any dead skin that covers the nipple opening.

2. Load $10-20 \mu \mathrm{l}$ of injection solution into a $50 \mu \mathrm{l}$ syringe with a $33 \mathrm{G}$ metal hub needle affixed. Sometimes, after injection, small amounts ( $1 \mu \mathrm{l}$ or less) can leak out of the nipple upon pulling out the needle. Therefore, it is recommended to inject 11 or $21 \mu$ respectively to account for the potential loss.

3. Hold the nipple gently with the fine tweezers and lift it slightly to position it for injection. It is not necessary to cut the nipple.

4. Inject the solution slowly to minimize potential damage caused by rapidly moving fluid within the ductal lumens. The injection rate should be maintained at approximately $40 \mu \mathrm{l} / \mathrm{min}$.

\section{Postoperative Care}

1. Observe the injection site. There should be no signs of trauma to the nipple region or surrounding tissue. Swelling in the area surrounding the nipple likely indicates a mammary fat pad injection rather than a successful intraductal injection.

2. Remove the animal from the nose cone and move to a separate cage for recovery. Place the cage under a heat lamp to prevent hypothermia and assist in recovery. Mice are housed singly and will be monitored closely until they regain consciousness and mobility.

\section{Analysis of Mammary Gland Tissue}

1. Upon completion of the study, mice are euthanized by cervical dislocation following $\mathrm{CO}_{2}$ compressed gas in an isolation chamber. Mammary glands are excised and may be used for whole mount preparation ${ }^{11}$, histology, or RNA and protein isolation.

\section{Representative Results}

The nipples can be easily localized using a stereomicroscope once hair has been removed in the area surrounding it (Figure 1A). To master the injection technique, it is recommended to inject Evans blue dye and monitor the integrity of the gland (Figures 1B and C). This approach also allows for the determination of appropriate volumes to be injected into each gland as one can visually assess whether the dye reaches the entire ductal system (Figures 1C and 2A). An image of the injected Evans blue image can then be compared to the whole-mounted mammary gland, in which the entire ductal tree is stained with Carmine alum dye (Figure 2B).

Please note that robustness of this injection method is highly dependent on the operator. For example, perforating the duct will result in a mammary fat pad injection and injecting the solution too fast may damage the ductal epithelial cells and provoke an inflammatory response. Successful intraductal injection enables the localized drug delivery to the mammary gland and reduces non-specific side-effects often observed otherwise.

An example image of a gland injected with fluorescently tagged siRNA targeting cyclophilin (a non-essential gene) is shown in Figure 3 . 

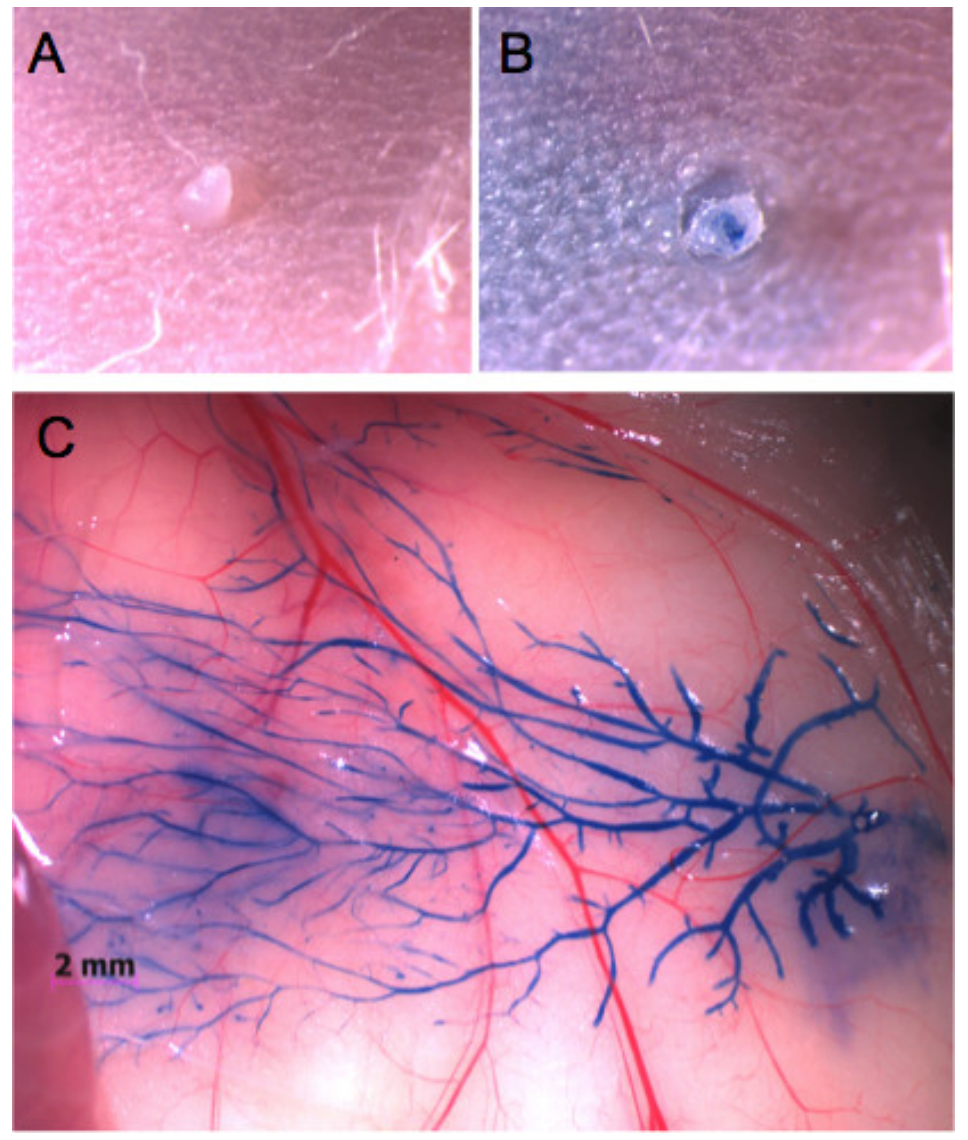

Figure 1. An inguinal mammary gland injected through the nipple with Evans blue dye. A) Appearance of the nipple before injection and B) after injection with $20 \mu \mathrm{l}$ Evans blue dye. No swelling or tissue damage is observed. C) Upon opening the skin, the dye permits visualization of the entire mammary ductal tree.
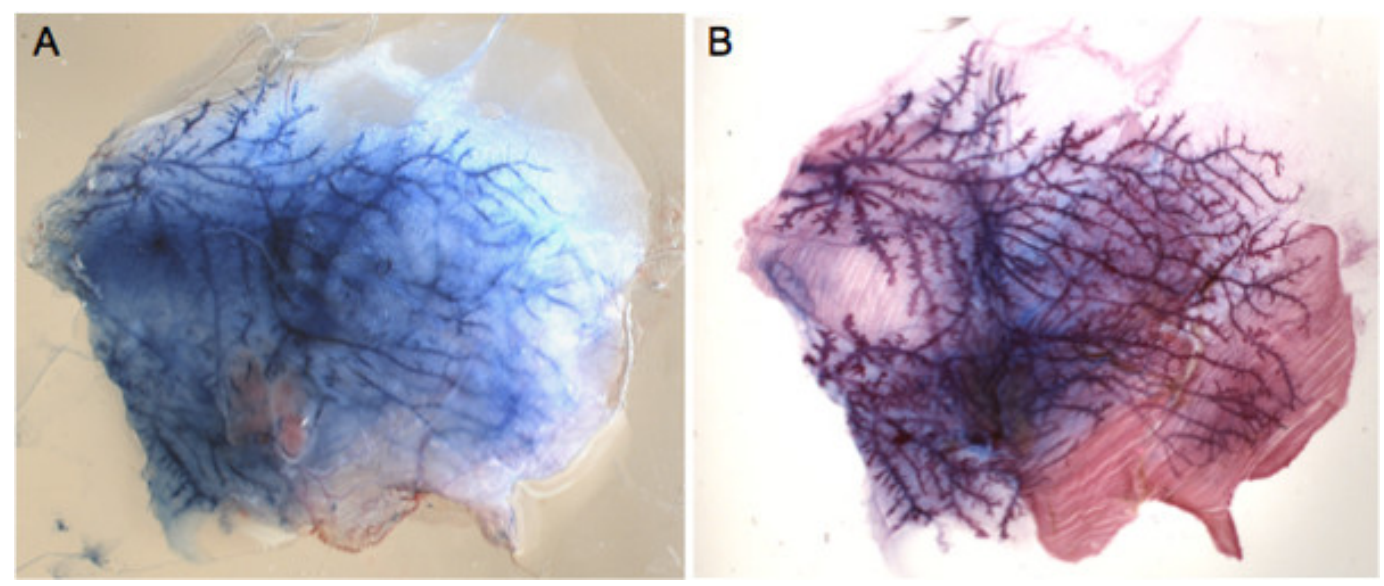

Figure 2. Whole-mount analysis of Evans blue-injected gland. A) Representative image of a dissected gland excised from the animal and spread onto a glass slide immediately after injection. B) Whole mount staining of the same gland with carmine dye after fat had been cleared. Comparison with A) confirms that the injected solution fills the mammary ductal tree and the gland is anatomically intact. 


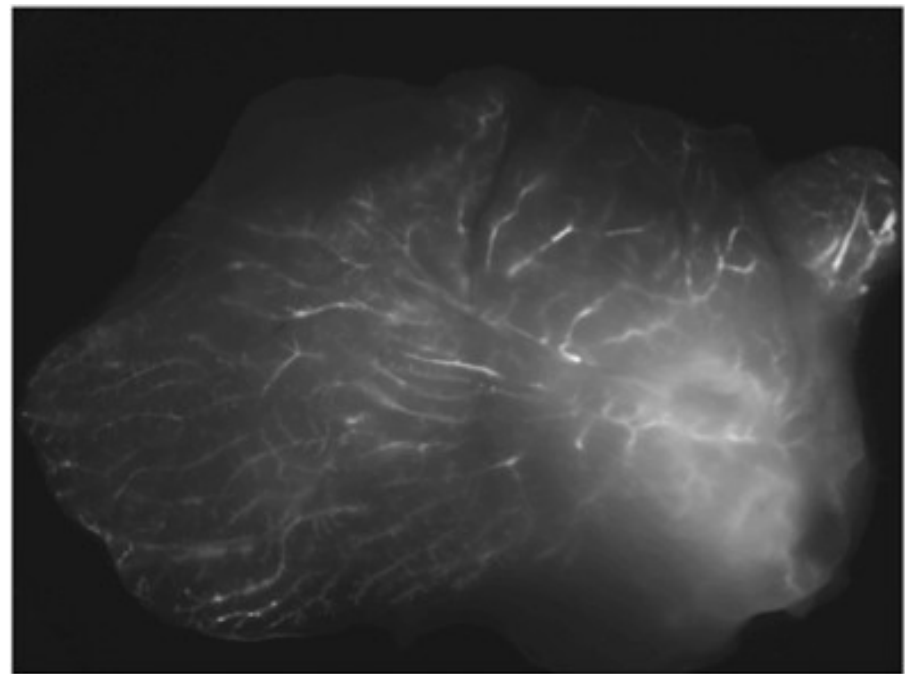

Figure 3. Intraductal delivery of fluorescent siRNA. Representative image of fresh tissue sample, excised $48 \mathrm{hr}$ postinjection.

\section{Discussion}

Transgenic and knockout mice are invaluable tools for studying the in vivo role of individual genes in breast cancer. However, it is costly and time-consuming to generate these animals and the gene knockdown is usually ubiquitous which sometimes precludes us from recognizing the specific effects of that gene on the mammary gland. Therefore, a targeted knockdown would alleviate issues of non-specific side-effects and toxicities in other organs.

The intraductal injection of siRNA presented here enables the localized gene knockdown in the mouse mammary gland. It is a rapid method for a targeted and localized drug delivery to the mammary gland not limited to siRNA delivery. Such a mammary gland-specific drug delivery avoids unspecific side-effects and toxicity to other organs and allows for the study of gene alterations at specific time points during mammary gland development and breast tumor formation. There is no evidence of siRNA particles in non-injected glands, the liver or other organs providing further evidence that this method presents a preferred way for localized gene knockdown in the mammary gland. Furthermore, the intraductal injections can be repeated weekly or biweekly over several months to allow for long-term monitoring of therapeutic agents.

This technique opens up the possibility for assaying the intraductal delivery of various reagents to the mouse mammary ducts. Advances in localized delivery in well-characterized mouse models should accelerate the application of non-invasive, targeted therapeutic strategies in humans.

\section{Disclosures}

The authors have no financial disclosures.

\section{Acknowledgements}

The authors would like to thank Michael Goldberg for preparing stabilized siRNA solution and Heather Tobin for her assistance with the animals. This study was funded by DOD Innovator Award W81XWH-08-1-0659 to DEl. The postdoctoral training of SK was supported by the Susan G Komen foundation (KG101329). The authors would like to thank Kristin Johnson for preparation of the schematic.

\section{References}

1. Dooley, W.C., et al. Ductal lavage for detection of cellular atypia in women at high risk for breast cancer. J Natl Cancer Inst. 93, 1624-1632 (2001).

2. Jacobs, L., Sukumar, S., \& Stearns, V. Intraductal therapy for the prevention of breast cancer. Curr Opin Investig Drugs. 11, 646-652 (2011).

3. King, B.L. \& Love, S.M. The intraductal approach to the breast: raison d'etre. Breast Cancer Res. 8, 206, doi:bcr1410 [pii] $10.1186 /$ bcr1410 (2006).

4. Murata, S., et al. Ductal access for prevention and therapy of mammary tumors. Cancer Res. 66, 638-645, doi:66/2/638 [pii] 10.1158/0008-5472.CAN-05-4329 (2006).

5. Stearns, V., et al. Preclinical and clinical evaluation of intraductally administered agents in early breast cancer. Sci Transl Med. 3, 106ra108, doi:3/106/106ra108 [pii] 10.1126/scitransImed.3002368 (2011).

6. Flanagan, M., Love, S., \& Hwang, E.S. Status of Intraductal Therapy for Ductal Carcinoma in Situ. Curr Breast Cancer Rep. 2, 75-82, doi:10.1007/s12609-010-0015-3 (2010).

7. Nguyen, D.-A., Beeman, N., Lewis, M., Schaack, J., and Neville, M. C. In Methods in mammary gland biology and breast cancer research (ed Ip, M.M., and Asch, B.B.) 259-270 (Kluwer Academic/ Plenum Publishers, 2000). 
8. Behbod, F., et al. An intraductal human-in-mouse transplantation model mimics the subtypes of ductal carcinoma in situ. Breast Cancer Res 11, R66, doi:bcr2358 [pii] 10.1186/bcr2358 (2009).

9. $\mathrm{Bu}, \mathrm{W} ., \mathrm{Xin}, \mathrm{L} ., \mathrm{T}$ Toneff, M., Li, L., \& Li, Y. Lentivirus vectors for stably introducing genes into mammary epithelial cells in vivo. J Mammary Gland Biol Neoplasia. 14, 401-404, doi:10.1007/s10911-009-9154-4 (2009).

10. Reddy, J.P. \& Li, Y. The RCAS-TVA system for introduction of oncogenes into selected somatic mammary epithelial cells in vivo. J Mammary Gland Biol Neoplasia. 14, 405-409, doi:10.1007/s10911-009-9157-1 (2009).

11. Plante, I., Stewart, M.K., \& Laird, D.W. Evaluation of mammary gland development and function in mouse models. J. Vis. Exp.. e2828 [pii] 10.3791/2828 (2011). 